\title{
Decentralized time domain muting for interference mitigation in LTE-advanced heterogeneous networks
}

\begin{abstract}
User equipments (UEs) offloaded from the MeNBs to the PeNBs via cell range extension (CRE) bias in a co-channel deployment suffered severe interference. The severity of the downlink interference varies significantly with the change in the CRE bias. The baseline approach for Interference mitigation technique based on time domain muting (TDM) of resources by MeNBs used trial and error technique which is causing resource wastage and is practically not feasible. Proposed here is a Model for TDM based on estimated cell load conditions and symbol efficiency (SE) as metrics to determine the muting ratio of resources. System level simulation was conducted to validate the throughput performances and the MeNBs- PeNBs resource trade-offs of the proposed method. Compared to the baseline (centralized) approach, the proposed decentralized TDM algorithm exhibited optimal throughput performance and adapted to the change in CRE bias with better trade-offs.
\end{abstract}

Keyword: Cell range extension; Heterogeneous networks; Inter-cell interference coordination; Picocell; Time domain muting 